\title{
MR Imaging Evolution of Endoscopic Cranial Defect Reconstructions Using Nasoseptal Flaps and Their Distinction from Neoplasm
}

\author{
K.O. Learned, N.D. Adappa, J.Y.K. Lee, J.G. Newman, J.N. Palmer, and L.A. Loevner
}

\begin{abstract}
BACKGROUND AND PURPOSE: Endoscopic endonasal approach is the procedure of choice for the resection of ventral skull base neoplasms, with defect closure requiring multilayer reconstruction. This study evaluates the temporal MR imaging evolution of nasoseptal flaps and free grafts used in endoscopic skull base reconstruction.
\end{abstract}

MATERIALS AND METHODS: Sixty-nine follow-up brain MRIs of 22 patients who had endoscopic skull base reconstruction using 26 nasoseptal flaps combined with 8 collagen-matrix dural grafts, 10 fascia lata grafts, and 10 intracranial fat grafts were retrospectively reviewed. Temporal changes in signal intensity, enhancement, thickness, and the configuration of reconstructive layers were evaluated. Tissue with signal intensity or enhancement different from that of normal evolving reconstructive layers at the surgical bed was evaluated, and its association with clinically confirmed tumor was assessed with the Fisher exact test.

RESULTS: All normal reconstructive layers were retracted to cranial defects and showed maturation of imaging features within 2-6 months. The immediate postoperative T2-isointensity to brain and enhancement of nasoseptal flaps persisted, but the flap thickness was reduced by $20 \%-30 \%$ (average thickness, $4.5 \pm 1.3 \mathrm{~mm}$ ); additionally, the $\mathrm{C}$ shape and vascular pedicle of the nasoseptal flaps became indistinct, but the flap location remained unchanged. The initial appearance of the nonenhancing fascia lata with variable T2 signal intensity became enhancing with increasing T2-hypointensity and a graft-thickness reduction of $\geq 50 \%$ (average thickness, $3.5 \pm 1.6 \mathrm{~mm}$ ). All fat grafts showed progressive resorption. In 6 patients, abnormal tissue represented residual or recurrent tumor $(P=.0001)$.

CONCLUSIONS: Maturation and stability of multilayer endoscopic skull base reconstructions on MR imaging occurs within 2-6 months postoperatively. Understanding of the normal imaging evolution of endoscopic skull base reconstructions is essential to distinguish them from neoplasms.

ABBREVIATIONS: $\mathrm{ASB}$ = anterior skull base; $\mathrm{CSB}$ = central skull base; $\mathrm{EEA}=$ endoscopic endonasal approach; $\mathrm{ESBR}$ = endoscopic skull base reconstruction; $\mathrm{NSF}=$ nasoseptal flap

$\mathrm{T}$ he traditional open craniofacial surgical approach to the ventral skull base allows a wide exposure, but at the cost of craniotomy, brain and cranial nerve retraction, and facial incision. The endoscopic transsphenoidal adenohypophysectomy is the standard in pituitary surgery. More recently, the use of the endoscopic endonasal approach (EEA) has expanded beyond the sella, and it

Received August 20, 2013; accepted after revision November 6.

From the Departments of Radiology (K.O.L., L.A.L.), Neurosurgery (J.Y.K.L.), and Otorhinolaryngology-Head and Neck Surgery (N.D.A., J.G.N., J.N.P., L.A.L.), University of Pennsylvania School of Medicine, Philadelphia, Pennsylvania.

Paper previously presented as a scientific oral presentation at: Annual Meeting of the American Society of Neuroradiology and the Foundation of the American Society of Neuroradiology Symposium, May 18-23, 2013; San Diego, California.

Please address correspondence to Kim O. Learned, MD, Department of Radiology, Neuroradiology Division, Hospital of the University of Pennsylvania, 219 Dulles Building, 3400 Spruce St, Philadelphia, PA 19104; e-mail: Kim.Learned@uphs.upenn.edu

三 Indicates article with supplemental on-line tables.

http://dx.doi.org/10.3174/ajnr.A3853 is now routinely used for the resection of a spectrum of sinonasal, ventral skull base, and intracranial pathologies. ${ }^{1-6}$ Endoscopic skull base reconstruction (ESBR) with multilayer closure of the cranial base defect using vascularized tissues has become the repair method of choice in EEA, resulting in low CSF leak rates comparable with those in the open surgical approach. ${ }^{7-10}$ The workhorse of ESBR in EEA is the vascularized pedicled nasoseptal flap (NSF), which is based on the nasoseptal branch of the sphenopalatine artery (Fig $1 A,-B) .{ }^{11,12} \mathrm{~A}$ combination of intradural fat graft, inlay subdural collagen matrix, and/or the fascia lata with onlay NSF is effective in repairing cranial defects (Fig 1C). ${ }^{7-10}$ Most cranial defects are sufficiently closed by a single NSF elevated from one side of the nasal septum. Larger, extended cranial defects may require harvesting of bilateral nasoseptal flaps from both sides of the nasal septum and additional free mucosal grafts to cover the entire defect.

Kang et $\mathrm{al}^{13}$ described the MR imaging appearance of ESBR 

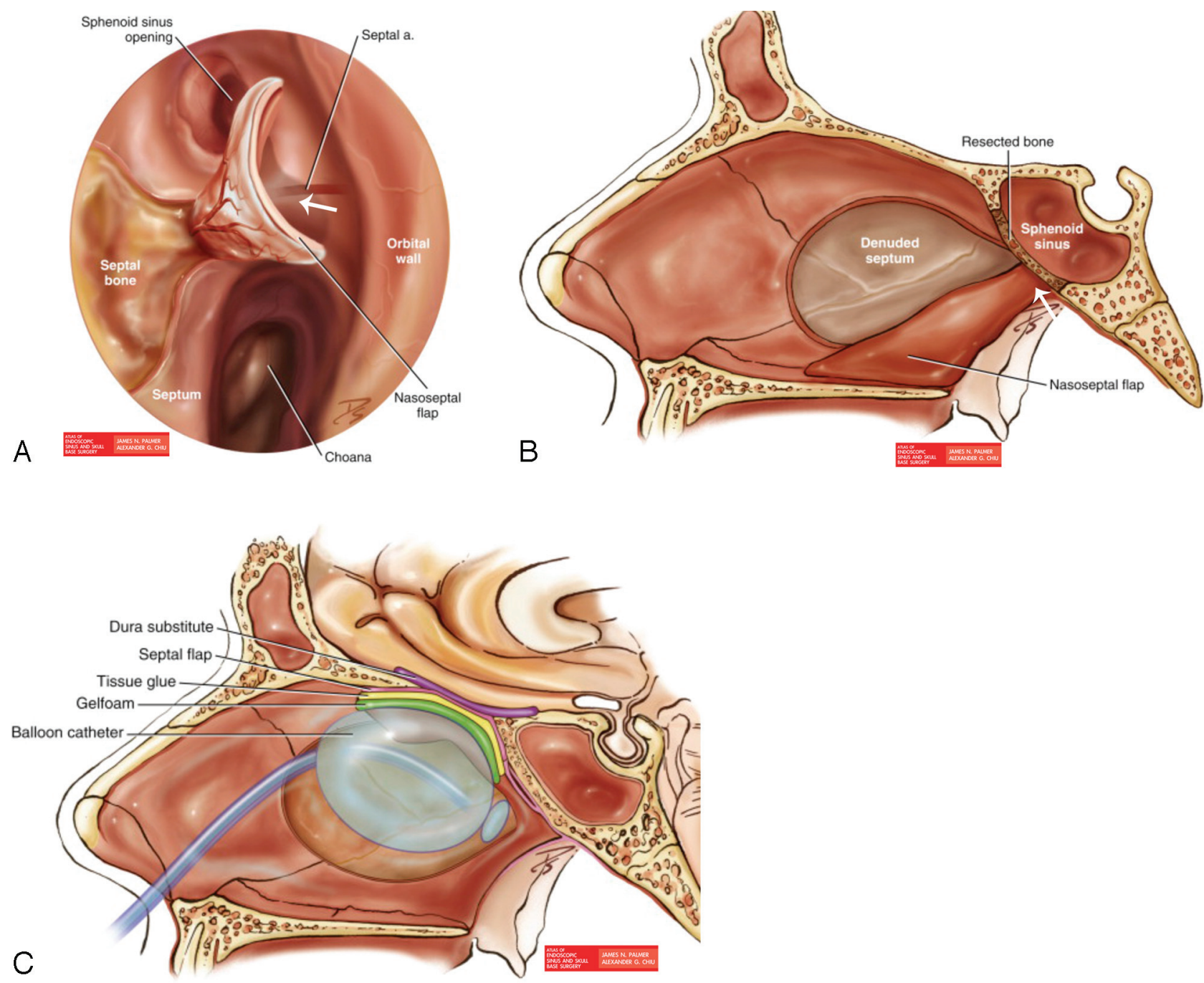

FIG 1. Multilayer ESBR using NSF. A, Endoscopic coronal view of the nasal cavity illustrates harvesting of the NSF from the nasal septum with the preserved septal artery vascular pedicle (arrow) arising from the sphenopalatine artery. B, Sagittal view of the harvested NSF shows that the flap is anchored by its pedicle (arrow), leading toward the ipsilateral sphenopalatine foramen. $C$, Sagittal drawing of the ESBR shows inlay dura substitute (such as collagen matrix, fascia lata) and onlay mucosal closure with an NSF (labeled as a septal flap). Autologous fat graft may be placed above the dura substitute layer to fill the intracranial resection bed. The NSF is held in place by biologic tissue glue. Reconstruction is bolstered with nasal sponges and/or a balloon catheter to prevent dislodging during the immediate postoperative period. Reprinted with permission from Atlas of Endoscopic Sinus and Skull Base Surgery. Palmer JN, Chiu AG. Copyright Elsevier, 2013. ${ }^{12}$

using NSFs to repair the surgically created skull base defects in a small cohort of patients following transsphenoidal resection of pituitary adenomas. ${ }^{13}$ Recently, Learned et al ${ }^{14}$ reported the immediate postoperative MR imaging evaluation of ESBR utilizing NSFs in a spectrum of sinonasal and skull base tumors resected using EEAs with defects along the entire ventral skull base. In the immediate postoperative period, NSFs have a C-shaped configuration on coronal and/or sagittal planes and avid enhancement, with the associated free grafts closely abutting the NSFs and showing no enhancement. ${ }^{13,14}$ Follow-up MR imaging is routinely performed for neoplastic surveillance in patients following EEA skull base resections and reconstructions. The normal evolution of the multilayer reconstructions following EEA is not well understood and can be mistaken for recurrent or persistent tumor. This study evaluates the temporal MR imaging appearances of grafts and NSFs used in ESBR.

\section{MATERIALS AND METHODS \\ Subjects}

Our institutional review board approved the retrospective review of our skull base surgery data base from January 2008 to May 2013 to identify 22 patients ( 13 men, 9 women; mean age, 54 years) who had undergone EEA skull base surgery, multilayer ESBR using NSFs, and brain MR imaging surveillance within 48 hours following surgery and thereafter for follow-up. The spectrum of pathologies included sinonasal tumors (36\%), clival chordomas and chondrosarcomas (18\%), and intracranial neoplasms (46\%). Surgically created skull base defects included anterior skull base (ASB) (41\%), central skull base (CSB) (36\%), and clival defects (23\%). In 22 ESBRs, 26 NSFs ( 18 single and 8 bilateral flaps) were used with a combination of 8 collagen matrix dural grafts (DuraGen; Integra Life Sciences, Plainsboro, New Jersey; Surgisis Biodesign Dural graft; Cook Medical, Bloomington, Indiana), 10 
Table 1: Patient clinical data

\begin{tabular}{|c|c|c|c|c|}
\hline $\begin{array}{c}\text { Pathologies (No. of Patients and } \\
\text { Average Tumor Size) }\end{array}$ & $\begin{array}{l}\text { Skull Base } \\
\text { Defects }\end{array}$ & $\begin{array}{c}\text { ESBR Tissues } \\
\text { (No. of Patients) }\end{array}$ & $\begin{array}{l}\text { Length of MRI } \\
\text { Follow-Up (mo) }\end{array}$ & $\begin{array}{l}\text { Adjuvant Therapy } \\
\text { (No. of Patients) }\end{array}$ \\
\hline Esthesioneuroblastoma $(n=6 ; 2.5 \mathrm{~cm})$ & ASB & $\begin{array}{l}\text { F, FL, NSF, FM }(n=1) \\
\text { D, NSF }(n=3) \\
\text { D, NSF, FM }(n=2)\end{array}$ & $9-32$ & $\begin{array}{l}\text { Radiation }(n=4), \\
\text { Chemoradiation }(n=2)\end{array}$ \\
\hline $\begin{array}{l}\text { Sinonasal endocrine carcinoma } \\
\qquad(n=1 ; 4.0 \mathrm{~cm})\end{array}$ & ASB & F, FL, NSF, FM $(n=1)$ & 16 & Chemoradiation $(n=1)$ \\
\hline $\begin{array}{l}\text { Sinonasal undifferentiated carcinoma } \\
\qquad(n=1 ; 2.2 \mathrm{~cm})\end{array}$ & ASB & $\mathrm{D}, \mathrm{NSF}(n=1)$ & 3 & Chemoradiation $(n=1)$ \\
\hline Chondrosarcoma $(n=1 ; 2.5 \mathrm{~cm})$ & Clivus & $\mathrm{D}, \operatorname{NSF}(n=1)$ & 21 & Radiation $(n=1)$ \\
\hline Chordoma $(n=3 ; 2.0 \mathrm{~cm})$ & Clivus & $\begin{array}{l}\operatorname{NSF}(n=2) \\
\text { F, FL, NSF }(n=1)\end{array}$ & $9-21$ & Radiation $(n=2)$ \\
\hline Meningioma $(n=4 ; 2.2 \mathrm{~cm})$ & $\begin{array}{l}\text { ASB } \\
\text { CSB } \\
\text { Clivus }\end{array}$ & $\begin{array}{l}\mathrm{D}, \mathrm{NSF}(n=1) \\
\mathrm{F}, \mathrm{FL}, \mathrm{NSF}(n=2) \\
\mathrm{F}, \mathrm{FL}, \operatorname{NSF}(n=1)\end{array}$ & $3-41$ & Radiation $(n=1)$ \\
\hline Craniopharyngioma $(n=1 ; 3.5 \mathrm{~cm})$ & CSB & $\mathrm{F}, \mathrm{FL}, \mathrm{NSF}(n=1)$ & 6 & \\
\hline Pituicytoma $(n=1 ; 3.1 \mathrm{~cm})$ & CSB & $\mathrm{F}, \mathrm{FL}, \mathrm{NSF}(n=1)$ & 13 & Radiation $(n=1)$ \\
\hline $\begin{array}{l}\text { Pituitary macroadenoma } \\
\qquad(n=4 ; 3.4 \mathrm{~cm})\end{array}$ & CSB & $\begin{array}{l}\operatorname{NSF}(n=1) \\
\text { F, NSF }(n=1) \\
\text { FL, NSF }(n=1) \\
\text { F, FL, NSF }(n=1)\end{array}$ & $6-27$ & Radiation $(n=1)$ \\
\hline
\end{tabular}

Note:-ASB indicates cribriform plates, crista galli, and/or ethmoid fovealis; CSB, planum sphenoidale, tuberculum sella, sella, and/or dorsum sella; $D$, collagen matrix dural graft; F, intracranial fat; FL, fascia lata; FM, free mucosa.

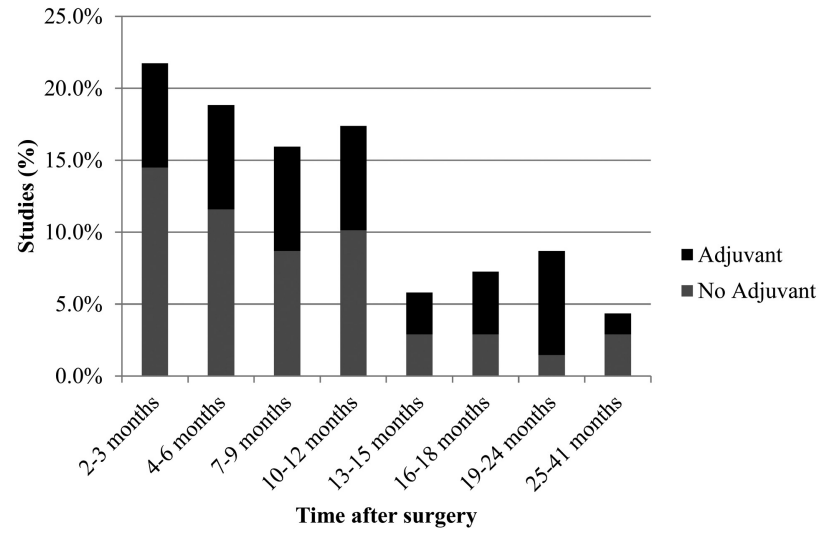

FIG 2. Graft shows the percentage of follow-up MR imaging studies performed before or without adjuvant treatment (gray bar) and after adjuvant treatment (black bar) versus postoperative time intervals.

autologous fascia lata grafts, 10 intracranial fat grafts, and 4 additional free mucosal grafts. Table 1 summarizes patient clinical data.

\section{Imaging Methods}

Sixty-nine follow-up MRIs were obtained at 2- to 6-month intervals, depending on tumor histology, adjuvant therapy, clinical status, and surveillance paradigm. The length of follow-up ranged from 3 to 41 months, with an average of 16.4 months. We categorized the follow-up MRIs of the entire cohort according to the time at which they were obtained after surgery: first follow-up at 2-3 months; subsequently, every 3-month-interval follow-up during the first 1-2 years after surgery; and every 6-month-interval follow-up thereafter. The graph in Fig 2 summarizes the percentage of serial follow-up MRIs after surgery and adjuvant treatment.

Brain MRIs were performed at 1.5T $(n=55)$ and 3T $(n=14)$ (Signa and Optima; GE Healthcare, Milwaukee, Wisconsin; and Espree, Avanto, Verio and Skyra; Siemens, Erlangen, Germany), including thin-section images of the sella turcica/ventral skull base with parameters detailed in On-line Tables 1 and 2. All patients received intravenous administration of $0.1-\mathrm{mmol} / \mathrm{kg}$ gadolinium (MultiHance; Bracco Diagnostics, Princeton, New Jersey).

\section{Imaging and Statistical Analysis}

All images were reviewed together in consensus by 2 neuroradiologists subspecialized in head and neck imaging. The NSFs and free grafts were evaluated for temporal changes in T2 signal intensity (in comparison with the gray matter and white matter of the brain parenchyma), the presence of enhancement, and the average thickness. The C-shaped configuration of enhancing NSFs in our cranial base reconstructions was best demonstrated on an enhanced sagittal and/or coronal plane. The free grafts were located above the enhancing NSFs in multilayer ESBRs. Therefore, the average of 2 maximal flap and graft thickness measurements at 2 different locations on enhanced sagittal and/or coronal planes was recorded. All studies of each patient were evaluated at the same time in our retrospective study to assess the temporal change of ESBRs. Imaging assessment was correlated with surgical techniques and clinical follow-up. The presence of tissue with signalintensity or enhancement different from that of normal evolving reconstructive layers was also evaluated, and its MR imaging characteristics were compared with those of the neoplasm on preoperative MR imaging. The association of the abnormal tissue that was similar to the preoperative neoplasm and clinically confirmed tumor was assessed with the Fisher exact test, using the Statistical Package for the Social Sciences, Version 20.0 (IBM, Armonk, New York).

\section{RESULTS}

All normal reconstructions demonstrated retraction of the reconstructive layers to the cranial defects with reduction in thickness and showed maximal alterations of the imaging features within 2-6 months, with little or no further change on subsequent imaging. Fifty-five percent of follow-up MR imaging studies were performed before or without adjuvant treatment and 45\%, af- 

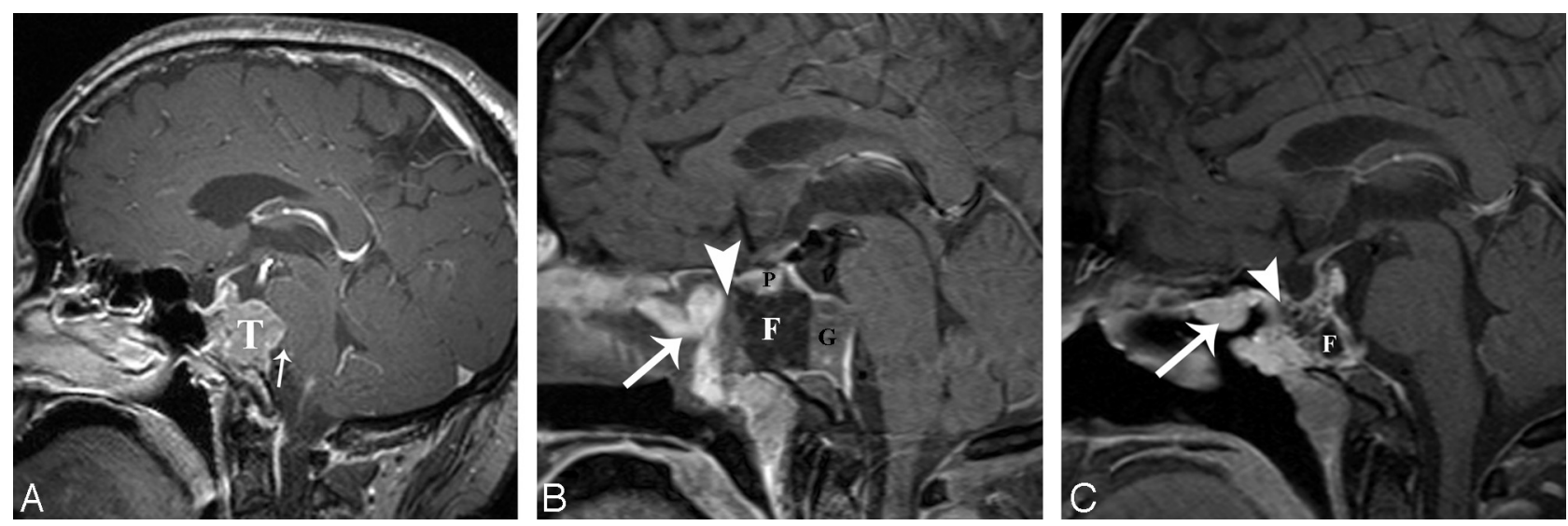

FIG 3. A 47-year-old man with normal evolution of the ESBR after EEA gross total resection and adjuvant radiation of a clival chordoma. A, Preoperative sagittal enhanced TIWI shows tumor (T) eroding the upper clivus and compressing the ventral pons (arrow). B, Immediate postoperative sagittal enhanced fat-saturated TIWI shows an enhancing C-shaped NSF covering the clival defect (arrow), abutting the nonenhancing fascia lata (arrowhead) and clivectomy fat packing (F). Nonenhancing Gelfoam (Phadia, Uppsala, Sweden) and blood (G), enhancing pituitary (P). C, Five-month follow-up sagittal enhanced fat-saturated TIWI shows a solidly enhancing slightly thinner NSF and retraction of the reconstruction with the enhancing fascia lata (arrowhead) inseparable from granulation tissue/resorbing fat (F). Marked nodularity along the anterior and inferior portions of the NSF relates to retraction and incorporation of the flap into the newly enhancing free grafts and granulation tissues.

ter adjuvant treatment (Fig 2). The patients' sinonasal inflammatory mucosal changes and adjuvant radiation or chemoradiation had no appreciable effect on the normal imaging evolution of ESBRs (Figs 3 and 4). The appearance of the different components of the flaps and their changes with time to become stable within 2-6 months are summarized in Table 2 and presented in detail below.

The immediate postoperative $\mathrm{T} 2$-isointensity to the brain (mostly similar to gray matter) and solid enhancement of 21 NSFs persisted, and the flap thickness was reduced by $20 \%-30 \%$ (average thickness, $4.5 \pm 1.3 \mathrm{~mm}$ ) (Figs 3 and 4 ). Their immediate postoperative distinctive $\mathrm{C}$ shape and vascular pedicle configurations became less well-defined on follow-up imaging as the NSFs incorporated into the newly enhancing free grafts and granulation tissues, but the flap location remained unchanged (Fig 3). The markedly nodular appearance of the NSF was noted in 4 of our patients (18\%) who did not have tumor recurrence (Fig 3C).

Five NSFs without enhancement on immediate postoperative MR imaging demonstrated 1- to 5-mm-thick solid enhancing tissue on follow-up. Similarly, 4 free mucosal grafts without enhancement on immediate postoperative MR imaging showed 1to 5-mm-thick solid enhancement on follow-up. Their enhancement correlated to clinically evident healing granulation tissue and/or mucosalization at the graft sites.

The immediate postoperative nonenhancing fascia lata with variable T2 signal intensity became mildly enhancing with increasing T2-hypointensity, and the graft thickness decreased by $\geq 50 \%$ (average thickness, $3.5 \pm 1.6 \mathrm{~mm}$ ) (Figs 3 and 4 ). The collagen matrix dural grafts remained nonvisible to $\leq 2 \mathrm{~mm}$, and the immediate postoperative nonenhancing thin grafts became inseparable from enhancing granulation tissue at the reconstructed skull base on follow-up imaging (Fig 5). All fat grafts showed progressive resorption with $\geq 50 \%$ reduction in volume within 2-6 months and near disappearance by 14 months (Figs 3 and 4$)$.

The nodular tissue with variable T2 signal intensity and en- hancement different from the normal evolution of reconstructive layers was seen at the interface of the reconstruction and tumor bed in 6 patients ( 1 sinonasal carcinoma, 1 chordoma, 1 meningioma, and 3 pituitary tumors) (Fig 5 ). The MR imaging characteristics of the nodular tissue were similar to those of the primary neoplasm seen on the preoperative MR imaging. In the patients with sinonasal carcinoma, recurrence occurred at the margin of the radiation field. The presence of the abnormal tissue was associated with neoplastic residua in 5 patients and recurrence in 1 patient $(P=.0001)$.

\section{DISCUSSION}

\section{Endoscopic Skull Base Surgery and Cranial Defect Reconstruction}

The endonasal approach provides a direct and less invasive surgical corridor to primarily midline sinonasal, skull base, and intracranial neoplasms along the ventral cranial base, with equivalent or better gross total resection compared with open surgery. ${ }^{1-7}$ Open skull base surgery, classically in the form of combined craniotomy and a transfacial or endonasal endoscope-assisted approach, is repaired with the vascularized pericranial flap routinely sutured to the edge of the dural defect. Cranial defect closure in EEA has unique challenges, including the inability to suture the reconstructive layers and the difficulty in using a pericranial flap. A variety of biologic glues and temporary supportive nasal bolsters has been used to secure the ESBR layers to the cranial defect, while awaiting granulation and incorporation of the reconstructive tissues into the surgical bed. ${ }^{8-10}$ The small dural defects have been successfully closed with nonvascularized free grafts such as seen in pituitary surgery. With the increasing size of cranial defects in EEA for resection of larger tumors and with deeper intra-arachnoid dissections and increased exposure to the cisterns, repair techniques using nonvascularized free grafts have shown unacceptable postoperative CSF leak rates. ${ }^{7,8}$ To allow maximal resection with CSF leak rates similar to those in open surgical approaches, ESBR in the EEA routinely necessitates 

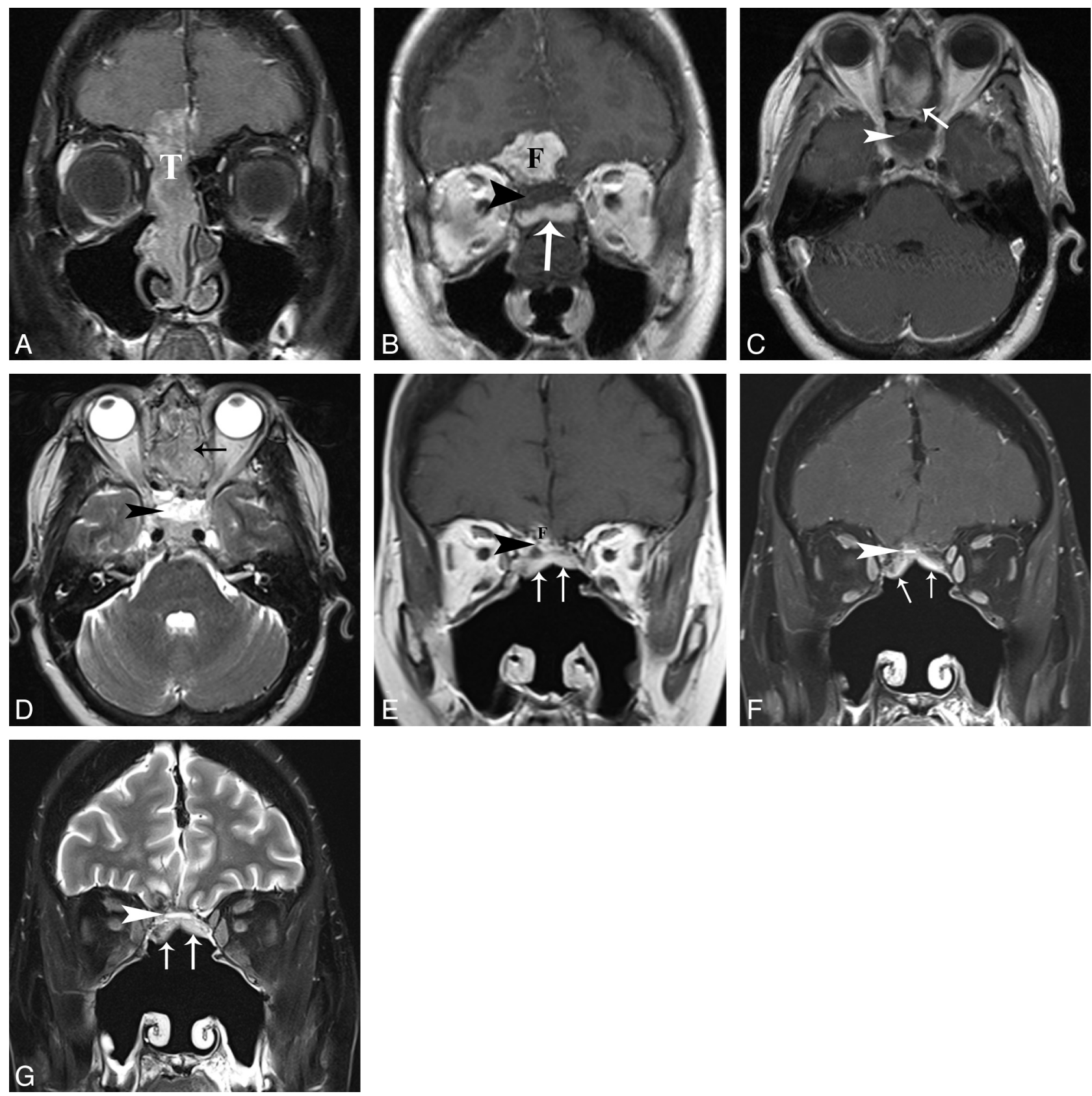

FIG 4. A 58-year-old woman status post transcribriform resection and adjuvant chemoradiation for sinonasal neuroendocrine carcinoma and NSF misinterpreted as a neoplasm. A, Preoperative coronal enhanced fat-saturated TTWI shows enhancing tumor (T) with intracranial extension. $B$ and C, Immediate postoperative coronal and axial enhanced TIWI shows fat (F) in the subfrontal bed, nonenhancing TI-hypointense fascia lata (arrowheads) at the bone defect, and mucosal closure with enhancing NSF (arrows). D, Immediate postoperative axial T2WI shows T2hyperintense fascia lata (arrowhead) and T2-isointense NSF (arrow). E, Three-month follow-up coronal enhanced TIWI shows normal evolution of the ESBR with retraction to the defect; significant resorption of intracranial fat graft (F); and a thin enhancing fascia lata (arrowhead) above the solidly enhancing NSF (arrows), mistaken for neoplasm in the official report. F and G, Sixteen-month follow-up ( 3 months after completion of chemoradiation) coronal fat-saturated enhanced TIWI and T2WI show little change of the enhancing fascia lata (arrowheads) and solidly enhancing T2-isointense NSF (arrows).

multilayer reconstruction using a vascularized NSF. The NSF is a pedicled vascular flap based on the posterior septal artery, a branch of the sphenopalatine artery. ${ }^{11}$ This robust vascularized flap has become the mainstay in ESBR, with reported postoperative CSF leak rates in 5\%-10\% of cases, a range similar to that in open procedures. ${ }^{7,8}$

Our ESBRs were performed in a multilayer stepwise fashion, beginning intracranially and advancing out to the sinona- sal mucosa, using a combination of autologous fat filling the intracranial surgical bed, subdural inlay collagen matrix underneath the edges of the dural defect and/or inlay-onlay fascia lata, and the final mucosal onlay closure with single or bilateral NSFs (Fig 1). Occasionally, additional free mucosal grafts taken from the nasal septum or turbinates were required to cover the far anterior edge of the cribriform defects, which were not reachable by the pedicled NSF (Table 1). 
Table 2: MR imaging evolution of ESBR from the immediate postoperative period to stability on follow-up

\begin{tabular}{|c|c|c|}
\hline & Immediate Postoperative MRI & Follow-Up MRI at 2-6 Months \\
\hline NSF & $\begin{array}{l}\text { Solid enhancement, T2-isointensity to brain (mostly } \\
\text { similar to gray matter), distinctive C shape and } \\
\text { vascular pedicle configurations }\end{array}$ & $\begin{array}{l}\text { Persistent solid enhancement, persistent T2-isointensity to brain } \\
\text { (mostly similar to gray matter), thickness reduction } \\
\text { by } 20 \%-30 \% \text {, average thickness of } 4.5 \mathrm{~mm} \\
\text { Less well-defined C shape and vascular pedicle configurations, } \\
\text { unchanged flap location }\end{array}$ \\
\hline Fascia lata & Nonenhancing, variable T2 signal intensity & $\begin{array}{l}\text { Mildly enhancing, increasing T2-hypointensity, thickness } \\
\text { reduction by } \geq 50 \% \text {, average thickness of } 3.5 \mathrm{~mm}\end{array}$ \\
\hline $\begin{array}{l}\text { Collagen matrix } \\
\text { dural graft }\end{array}$ & Nonenhancing, nonvisible to $\leq 2 \mathrm{~mm}$ & $\begin{array}{l}\text { Inseparable from enhancing granulation tissue at the } \\
\text { reconstruction site, nonvisible to } \leq 2 \mathrm{~mm}\end{array}$ \\
\hline Fat graft & Fat signal intensity fills intracranial surgical bed & $\begin{array}{l}\text { Fat signal intensity in retracted intracranial surgical bed, } \\
\text { progressive resorption with volume reduction by } \geq 50 \%\end{array}$ \\
\hline
\end{tabular}


FIG 5. A 52-year-old man following transplanum resection and chemoradiation for sinonasal undifferentiated carcinoma has abnormal evolution of the ESBR, consistent with recurrent tumor. A, Preoperative coronal fat-saturated T2WI shows tumor (T) invading the planum sphenoidale and right optic canal (arrowhead). S indicates sinus secretions. $B$ and C, Immediate postoperative coronal and axial enhanced fat-saturated TTWIs show an enhancing NSF (arrows) at the planum-sphenoidale defect with a right-sided vascular pedicle (asterisk). The thin nonenhancing collagen matrix dural inlay above the NSF is indiscernible. The nasal cavity is packed with sponge. $D$ and $E$, Three-month follow-up coronal fat-saturated T2WI and enhanced TIWI show retraction of the enhancing NSF (arrows) into the skull base defect with a stable location of the right pedicle seen on the axial image (not shown) and mildly enhancing T2-hypointense dural graft/granulation (bow arrows). Nodular tumor recurrence at the edge of the reconstruction in the left sphenoid sinus ( $T$ ) and along the supraorbital dura (arrowheads) has signal intensity and mild enhancement similar to that in preoperative tumor and different from that in the adjacent reconstructive layers.

\section{MR Imaging Follow-Up of Endoscopic Skull Base Reconstruction}

The imaging features of ESBR with the characteristic enhancing $\mathrm{C}$ shape and vascular pedicle configuration of the NSF and nonenhancing free grafts on immediate postoperative MR imaging have been described previously. ${ }^{13,14}$ Kang et $\mathrm{al}^{13}$ reported NSFs used to reconstruct defects following resection of pituitary adenomas and found stable signal intensity but variable changes in thickness and enhancement of NSFs on follow-up MRI at 3-7 months. ${ }^{13}$ They described 2 flaps without enhancement in the immediate postop- erative period that showed delayed enhancement on follow-up imaging and 8 flaps that enhanced in the immediate perioperative period with persistent enhancement in $7 / 8$ flaps on follow-up imaging. Five of the initially enhancing NSFs became thinner; 2, thicker; and the average flap thickness varied from nonvisualization to $7 \mathrm{~mm}$. They also noted reduction in the thickness of the multilayer reconstruction between the graft and defect. Their cohort of pituitary adenomas did not require adjuvant chemoradiation. 
Our long-term follow-up (range, 3-41 months; average, 16.4 months) showed some differences from the published results of Kang et al. ${ }^{13}$ None of our enhancing NSFs lost their immediate postoperative solid enhancement, and all demonstrated mild reduction in thickness. Our average flap thickness of $4.5 \mathrm{~mm}$ was comparable. None of our cases, including a similar cohort with a transellar approach without adjuvant treatment, had increases in flap thickness as seen in 2 patients in the study of Kang et al (an increase of 1 and $2 \mathrm{~mm}$ in flap thickness). This discrepancy may be explained by sampling error. During the immediate postoperative period, the NSF stood out as the only enhancing tissue among the nonenhancing free grafts and postoperative sinus contents. Therefore, its unique enhancing C-shaped configuration was easily delineated. Delayed enhancement of the free grafts, retraction and reduction in the thickness of the reconstructive layers, and integration of the enhancing NSF into the reconstruction site resulted in its less distinct configuration on follow-up MR imaging (Fig 3). The nonenhancing NSFs on immediate postoperative MR imaging probably compromised the vascular pedicles. Theoretically, they served as free mucosal grafts at the reconstructive site. Therefore, these nonenhancing NSFs behaved in a manner similar to those in the free mucosal grafts on follow-up; both appeared as thin enhancing granulation-mucosalization, radiographically and clinically.

The reduction in thickness of free grafts in our cohort varied. The fascia lata remained thicker than DuraGen, and its noticeable reduction in thickness probably reflects resolution of immediate postoperative swelling with increasing T2-hypointensity (Fig 4). Enhancement on follow-up reflects the incorporation of the fascia lata into the granulation tissue at the cranial defect. In reconstructions using only inlay subdural collagen matrix as the dural graft, the nonvisible to $\leq 2$-mm nonenhancing collagen matrix became indistinguishable from the enhancing granulation at the defect (Fig 5).

In addition, the stabilization of the mature imaging features occurred within 2-6 months following surgery, with little or no further change on subsequent imaging. Seventy-four percent of our follow-up MRIs were obtained within the first year after surgery, because the initial follow-up intervals were generally closer, with a resulting higher number of studies, especially those for oncologic surveillance. Close to equal proportions of the imaging studies were performed in patients after they received adjuvant chemo- and/or radiation treatment $(45 \%)$ and in patients who did not or have not received adjuvant treatment (55\%). Notably, the adjuvant radiation or chemoradiation and the sinonasal inflammatory changes had no appreciable effect on the normal imaging evolution of ESBRs in our oncologic patients (Fig 4).

Imaging studies evaluating postoperative endoscopic pituitary surgery demonstrated the involution of surgical materials and identification of residual-recurrent adenoma by location, characteristic signal intensity, and nodular enhancing patterns that were identical to those of the corresponding preoperative adenoma. ${ }^{15,16}$ Our evaluation of parasellar neoplasms following EEAs is concordant with those of MR imaging studies of transellar hypophysectomy. Our study also showed that the full-thickness enhancement of T2-isointense NSFs, which is different from the thin peripheral enhancement of the adjacent T2-hyperintense si- nonasal mucosa, may mimic residual or recurrent tumor in patients with sinonasal neoplasms if the radiologist is not familiar with the normal appearance and evolution of these reconstructions (Fig 4). To avoid this pitfall, proper identification of the normal NSF on imaging and evaluation of the reconstructive margins for the presence of nodular tissue with signal characteristics and enhancement similar to that of preoperative neoplasm and different from that of the reconstructive layers are paramount (Fig 5).

\section{Limitations and Implications for Patient Care}

Limitations of our study include its retrospective nature and small sample size limited to an adult population with predominantly midline neoplasms. Even though the scanner variability makes our findings applicable to most practices, its effects on our results are uncertain, and that uncertainty may explain some of the minor differences in our results compared with those of Kang et al. ${ }^{13}$ Our reported imaging features of free grafts were limited to collagen matrix dural grafts and the fascia lata, and different materials used at other institutions may appear different. However, these free tissue grafts are most commonly used at many centers. ${ }^{8-10}$ The vascularized NSF, the workhorse of ESBR in EEA, is always present irrespective of the type of free grafts and reconstruction techniques.

With the ongoing evolution of surgical navigation, endoscopic instrumentation, and the growing success of EEA and ESBR in achieving gross total resection and reliable closure similar to that in the open approach, EEA is becoming a surgical standard for carefully selected cases in the minimally invasive surgical arena. ${ }^{1}$ As these procedures are increasing across the country, neuroradiologists will increasingly face the challenge of postoperative surveillance following EEA and ESBR, which potentially have unique patterns of neoplastic residua and recurrence compared with those of traditional open surgery.

\section{CONCLUSIONS}

Understanding the normal imaging evolution of multilayer ESBR, specifically the persistent T2-isointensity with solid enhancement of the NSF and the maturation of the reconstructive layers within 2-6 month follow-up, is critical in the imaging assessment for tumor surveillance following an EEA to skull base neoplasms. Recognition of typical patterns of evolution should help the interpreting radiologist distinguish expected posttreatment change from tumor recurrence.

Disclosures: John Y.K. Lee-UNRELATED: Payment for Lectures (including service on Speakers Bureaus): Baxter. Jason G. Newman-UNRELATED: Stock/Stock Options: Visionsense; Other: Intuitive Surgical, Comments: proctor for transoral robotic surgery. Laurie A. Loevner-UNRELATED: Grants/Grants Pending: American College of Radiology Imaging Network, Payment for Lectures (including service on Speakers Bureaus): Continuing Medical Education, university visiting professorships, International Diagnostics Course in Davos, Greece, and Hong Kong, Royalties: books authored-Temporal Bone Imaging (Thieme), Brain Case Review (Elsevier). *Money paid to the institution.

\section{REFERENCES}

1. Castelnuovo P, Dallan I, Battaglia P, et al. Endoscopic endonasal skull base surgery: past, present and future. Eur Arch Otorhinolaryngol 2010;267:649-63

2. Harvey RJ, Winder M, Parmar P, et al. Endoscopic skull base surgery 
for sinonasal malignancy. Otolaryngol Clin North Am 2011;44: $1081-140$

3. Komotar RJ, Starke RM, Raper DM, et al. Endoscopic skull base surgery: a comprehensive comparison with open transcranial approaches. Br J Neurosurg 2012;26:637-48

4. Koutourousiou M, Gardner PA, Tormenti MJ, et al. Endoscopic endonasal approach for resection of cranial base chordomas: outcomes and learning curve. Neurosurgery 2012;71:614-24

5. Leng LZ, Greenfield JP, Souweidane MM, et al. Endoscopic, endonasal resection of craniopharyngiomas: analysis of outcome including extent of resection, cerebrospinal fluid leak, return to preoperative productivity, and body mass index. Neurosurgery 2012;70:110-23

6. Dehdashti AR, Ganna A, Witterick I, et al. Expanded endoscopic endonasal approach for anterior cranial base and suprasellar lesions: indications and limitations. Neurosurgery 2009;64:677-87

7. Kassam AB, Prevedello DM, Carrau RL, et al. Endoscopic endonasal skull base surgery: analysis of complications in the authors' initial 800 patients. J Neurosurg 2011;114:1544-68

8. Harvey RJ, Parmar P, Sacks R, et al. Endoscopic skull base reconstruction of large dural defects: a systematic review of published evidence. Laryngoscope 2012;122:452-59

9. Liu JK, Schmidt RF, Choudhry OJ, et al. Surgical nuances for nasoseptal flap reconstruction of cranial base defects with high-flow ce- rebrospinal fluid leaks after endoscopic skull base surgery. Neurosurg Focus 2012;32:E7

10. Kassam AB, Carrau RL, Snyderman CH, et al. Endoscopic reconstruction of the cranial base using a pedicled nasoseptal flap. Neurosurgery 2008;63:ONS44-52, discussion ONS52-53

11. Hadad G, Bassagasteguy L, Carrau RL, et al. A novel reconstructive technique after endoscopic expanded endonasal approaches: vascular pedicle nasoseptal flap. Laryngoscope 2006;116:1882-86

12. Palmer JN, Chiu AG. Atlas of Endoscopic Sinus and Skull Base Surgery. Philadelphia: Elsevier; 2013

13. Kang MD, Escott $\mathrm{E}$, Thomas $\mathrm{AJ}$, et al. The MR imaging appearance of the vascular pedicle nasoseptal flap. AJNR Am J Neuroradiol 2009;30:781-86

14. Learned KO, Adappa ND, Loevner LA, et al. MR imaging evaluation of endoscopic cranial base reconstruction with pedicled nasoseptal flap following endoscopic endonasal skull base surgery. Eur J Radiol 2013;82:544-51

15. Steiner E, Knosp E, Herold CJ, et al. Pituitary adenomas: findings of postoperative MR imaging. Radiology 1992;185:521-27

16. Yoon PH, Kim DI, Jeon P, et al. Pituitary adenomas: early postoperative MR imaging after transsphenoidal resection. AJNR Am J Neuroradiol 2001;22:1097-104 